\title{
La necesidad de una posición crítica en la investigación psicológica. Apuntes sobre teoría y experiencia*
}

\section{The Need for a Critical Position in Psychological Research. Notes on Theory and Experience}

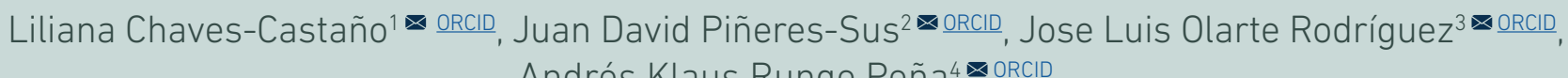
Andrés Klaus Runge Peña ${ }^{4} \underline{0 R C I D}$

Universidad de Antioquia

Colombia

Fecha correspondencia:

Recibido: octubre 9 de 2019.

Aceptado: mayo 7 de 2020.

Forma de citar:

Chaves-Castaño, L., Piñeres-

Sus, J. D., Olarte, J., \& Runge, A.

K. (2020). La necesidad de una posición crítica en la investigación psicológica. Apuntes sobre teoría y experiencia. Rev. CES Psico, 13(2), 166-179.

\section{Open access \\ (c) Copyright \\ Licencia creative commons \\ Etica de publicaciones \\ Revisión por pares \\ Gestión por Open Journal System DOl: http://dx.doi.org/10.21615/ cesp.13.2.11 \\ ISSN: 2011-3080}

Sobre el artículo:

* La reflexión que aquí se presenta hace parte de la tesis del Doctorado en Educación de Liliana Chaves

Comparte

\section{Resumen}

En el presente artículo argumentamos una tesis doble. La primera premisa sugiere que las distintas formas de investigación en psicología deben tomarse en serio el ejercicio de la crítica. La segunda premisa realiza una crítica racional del lugar que tienen teoría y experiencia en las prácticas investigativas. Algunos investigadores en psicología se encuentran en un doble riesgo: o bien parten de una concepción implícita de la experiencia según la cual ella no es útil para criticar las propias asunciones teóricas sino sólo para ilustrarlas; o bien, y en consecuencia, consideran también implícitamente que las teorías son dogmas cerrados que no se dejan someter a la crítica racional. Por esta razón, ponemos en duda escisiones entre investigadores, a propósito de la distinción entre investigación cualitativa y cuantitativa, teórica y empírica, entre otras. Argumentamos que, a pesar de sus esfuerzos por diferenciarse, los investigadores se parecen más de lo que estarían dispuestos a reconocer.

Palabras clave: Crítica, Experiencia, Investigación en Psicología, Metodología.

\section{Abstract}

In the article we argue a double thesis. The first premise suggests that the different forms of research in psychology should take the exercise of criticism seriously. The second premise makes a rational criticism when theory and experience take place in research practices. Some researchers are in a double risk: either part of an implicit conception of experience according to which it is not useful to criticize the theoretical assumptions but only to illustrate them, or it is also implicitly considered that theories are closed dogmas that cannot be rationally criticized. For this reason, we question divisions among researchers, for example, the distinction between qualitative and quantitative, theoretical and empirical research, etc. We argue that, despite their efforts to differentiate, researchers are more alike than they would be willing to acknowledge.

Keywords: Criticism, Experience, Methodology, Research in Psychology. 
Castaño, titulada: “El debate entre las teorías de la mente de dominio general y las teorías de la mente de dominio específico: su aporte a la reflexión antropológicopedagógica", dirigida por el Prof. Dr. Phil., Andrés Klaus Runge Peña.

Sobre los autores:

1. Doctorante en Educación. Magister. Profesora titular Departamento de Psicología, Universidad de Antioquia. Grupo de Investigación en Psicología Cognitiva de la Universidad de Antioquia. Grupo de Investigación sobre Formación y Antropología Pedagógica e Histórica -FORMAPH- de la Universidad de Antioquia.

2. PhD. Profesor asistente Departamento de Psicología, Universidad de Antioquia. Grupo de Investigación en Psicología Cognitiva de la Universidad de Antioquia.

3. Candidato a Magister en Psicología Universidad de Antioquia.

4. Doctor en Pedagogía. Profesor titular Facultad de Educación, Universidad de Antioquia. Coordinador del Grupo de Investigación sobre Formación y Antropología Pedagógica e Histórica -FORMAPH- de la Universidad de Antioquia.

\section{Consideraciones iniciales}

Pretendemos argumentar una tesis doble. La primera premisa sugiere que nuestras formas de investigación deben tomarse en serio aquello que, desde Kant (1781/2008), se conoce bajo el nombre de crítica. La segunda premisa realiza una crítica racional del lugar que tienen teoría y experiencia en nuestras prácticas investigativas. En no pocos casos, la investigación psicológica funciona dogmáticamente: utiliza la experiencia para ilustrar sus propias asunciones teóricas. Vista así, la experiencia no nos enseña nada pues no sirve para criticar nuestras creencias conceptuales. Como consecuencia, la teoría se mantiene intacta, al margen de todo cuestionamiento, a la manera de un dogma irrefutable.

Debemos aclarar, no obstante, que no nos referimos aquí a todos los investigadores de la disciplina psicológica. Al contrario, el primer tema de este artículo se concentra en la figura de Piaget, específicamente en su propia desconversión de la filosofía. Su relato constituye un ejemplo inmejorable del valor del científico, y de su justa utilización de teoría y experiencia. Leyendo a Piaget (1965/1970) nos hemos dado cuenta de la importancia de mantener viva nuestra memoria; cosas que le sucedían a él, hace ya un buen tiempo, continúan casi intactas en el presente. De allí que lo hayamos intitulado, El pasado como presente: algunos apuntes sobre la actualidad de un prejuicio.

Sin embargo, nuestros argumentos sí ponen en duda escisiones tan pronunciadas en nuestro medio entre investigadores, a propósito de la distinción entre investigación cualitativa y cuantitativa, teórica y empírica, entre otras. Argumentamos que, a pesar de sus esfuerzos por diferenciarse, frecuentemente se parecen más de lo que estarían dispuestos a reconocer. Unos y otros parecen descuidar el ejercicio racional de la crítica. Por consiguiente, el segundo tema tiene por título: La crítica como método. Finalmente, mostramos que teoría y experiencia tienden a ser utilizadas de manera dogmática en nuestras formas de investigación. De allí que hayamos pensado un Breve apunte sobre teoría y experiencia.

\section{El pasado como presente: algunos apuntes sobre la actualidad de un prejuicio}

Quisiéramos comenzar estas reflexiones trayendo al presente un fragmento de una historia narrada hace ya un buen tiempo por uno de los más grandes psicólogos y, valga decir, epistemólogos de la historia. Ahora bien, antes de concentrarnos en el relato mismo, un par de palabras acerca de la importancia del pasado pueden ser dichas. Siendo la memoria tan frágil, es nuestro deber recuperar aquellos momentos tanto teóricos como también vitales que, al haberse quedado en algún lugar del continuum de la historia de las ideas, en el mejor de los casos son considerados como pertenecientes a la prehistoria de nuestra disciplina. Dicho de otro modo, los confinamos a un pasado del cual no percibimos ningún nexo con nuestra situación presente. En suma, a los ojos del espectador actual la experiencia pasada aparece bajo dos modalidades, a saber: o bien constituye una pieza de museo únicamente interesante para el coleccionista; o bien no tiene importancia para el presente. Tal como es evidente, ambas modalidades no sólo denigran del pasado sino que, dicho de modo taxativo, no creen que en él exista una fuente de experiencia auténtica. En lo que sigue, vamos a partir de una premisa completamente contraria a la de aquellos que desprecian el pasado. Si es cierto que la argumentación puede avanzar gracias a lo que, desde Aristóteles, los lógicos llaman principio de contradicción, entonces debemos decir que el pasado tiene una importancia fundamental. Él aparece como el 
El relato aparece en "Sabiduría e llusiones de la Filosofía" de Piaget (1965/1970); entre muchas otras cosas, allí el autor narra cómo dejó de ser un filósofo interesado en la tradición metafísica y especulativa (tradición a la cual, dicho sea de paso, no trata nunca de modo denigrante) para convertirse en un psicólogo científico, en un epistemólogo genético y, habría que añadir, en un lógico especializado capaz de trabajar en el nivel de los argumentos sin descuidar el de los datos (sobre este asunto véase también Beth \& Piaget, 1961/1980; Piaget, 1957, 1971/1977; Smith, 2006a). fundamento de nuestro presente, cierto, aunque más específicamente él constituye nuestro presente (en una línea similar se encuentran los trabajos de Daniels, Cole. \& Wertsch, 2007; González, 2010; Müller, Carpendale, \& Smith, 2009; Overton, 1990; Rivière, 1985; Tryphon \& Vonèche, 1996/2000; Van der Veer, 2014; Yasnitsky, Van der Veer, \& Ferrari, 2014; Yasnitsky \& Van der Veer, 2016; Zavershneva \& Van der Veer. 2018).

Dicho lo anterior, teniendo como fondo la idea de que el pasado sigue siendo nuestro presente - una idea que pretende mantener viva la memoria (teórica y, claro está, disciplinar) - , nos referiremos ahora al fragmento de historia mencionado y, por supuesto, a su narrador. Se trata de una historia que, aunque pequeña en extensión, describe muy bien muchos de los prejuicios que todavía hoy son visibles; de su lectura se infiere que, sin importar el tiempo transcurrido, y a pesar incluso de nuestra ventaja histórica, hemos preferido dejar como incuestionadas nuestras asunciones ad hoc. El relato aparece en "Sabiduría e Ilusiones de la Filosofía" de Piaget (1965/1970); entre muchas otras cosas, allí el autor narra cómo dejó de ser un filósofo interesado en la tradición metafísica y especulativa (tradición a la cual, dicho sea de paso, no trata nunca de modo denigrante) para convertirse en un psicólogo científico, en un epistemólogo genético y, habría que añadir, en un lógico especializado capaz de trabajar en el nivel de los argumentos sin descuidar el de los datos (sobre este asunto véase también Beth \& Piaget, 1961/1980; Piaget, 1957. 1971/1977; Smith, 2006a).

Piaget (1965/1970) relata tres razones que lo llevaron a su progresiva desafección por los métodos tradicionales de la filosofía - aunque deja muy en claro que los problemas de la filosofía y la investigación empírica bien pueden ser similares: 1) A pesar de su interés por la filosofía, fue experimentando malestar por el modo de argumentación filosófico. Se percató de que, si bien la filosofía puede llevar al planteamiento de hipótesis sugerentes, habida cuenta de su capacidad especulativa, esas hipótesis necesitan ser comprobadas mediante un conjunto de hechos establecidos experimentalmente o mediante una deducción guiada por un algoritmo preciso, como en la lógica. 2) La segunda razón tiene que ver con la dependencia de las corrientes filosóficas de las transformaciones sociales y políticas. Las repercusiones en el movimiento de las ideas de las convulsiones sociales y políticas de su tiempo significaron, para Piaget, la posibilidad de poner en duda el valor objetivo y universal de las posturas filosóficas. 3) Por último, la tercera razón que, en las propias palabras de Piaget, lo llevó a su desconversión frente a la filosofía puede ser interpretada en términos de la progresiva inconmensurabilidad entre sus propios trabajos y el de sus antiguos colegas metafísicos. Leamos:

Esta tercera razón me fue advertida por la reacción de unos cuantos filósofos, cuyas interpretaciones y críticas me daban la impresión de que ya no hablábamos el mismo lenguaje; y no a causa de su actitud crítica (acabamos de ver que la crítica es una función esencial de la cooperación racional) sino porque entreveía una injerencia poco válida del juicio filosófico en el terreno de la investigación científica (Piaget, 1965/1970, p. 25).

Dos son los ejemplos que expresan esta tercera razón de su distanciamiento frente al trabajo del filósofo. Por un lado, el juicio filosófico busca prescribirle sus propias normas al científico. Y si bien Piaget reconoce que esta prescripción es una tendencia natural de la filosofía pues su función principal es la coordinación de los valores, no por ello está de acuerdo con la imposición normativa de su juicio, sobre la labor del 
Pág 169

Dicho llanamente, si bien pueden tener problemas similares, ciencia y filosofía se diferencian en su forma de trabajo. El metafísico trabaja por su cuenta, bajo el ideal del sistema filosófico - de allí que Piaget ubique a la lógica y a la epistemología por fuera de la disciplina filosófica (para ampliar esta afirmación véase Piaget, 1950/1975a, 1950/1975b; Smith, 2006b). Por su parte, el científico confía plenamente en la cooperación racional: su ambición no es el sistema aislado del trabajo de los otros (para una crítica de la noción de sistema filosófico véase especialmente los trabajos de Adorno, 1970/2005; Horkheimer \& Adorno, 1969/1998; Horkheimer, 1947/2010). científico, por parte del filósofo. En esto se encuentra muy cerca de los críticos de Popper, por ejemplo, Feyerabend (1975/2003) y Kuhn (1962/2013), para quienes la ciencia no funciona acomodándose a ninguna norma impuesta externamente. Dicho en otras palabras, el científico hace lo que puede hacer y no lo que otro le dice que debe hacer.

Por otro lado, esta tercera razón del distanciamiento de Piaget (1965/1970) frente a la tradición metafísica se apoya en otro ejemplo. Un ejemplo que, a todas luces, es mucho más importante para el problema que queremos hacer visible en este lugar; para ser precisos, él constituye el relato acerca del cual hemos querido escribir desde el principio. A través suyo, el psicólogo suizo nos dice que otra forma utilizada por el filósofo para injerir en la praxis del investigador es “(...) la de querer clasificarle a la fuerza" (Piaget, 1965/1970, p. 27). Su relato comienza así: "El filósofo I. Benrubi escribió una especie de reseña de conjunto sobre las corrientes filosóficas de habla francesa, y me hizo el honor de citarme, sin discutirme, pero clasificándome entre los positivistas" (p. 26). A pesar de que intentó persuadir a su interlocutor de lo contrario, Piaget no tuvo éxito en hacerle comprender sus diferencias con el positivismo. Verbigracia: ocuparse de los hechos positivos no significa ser positivista; en cuanto epistemología, el positivismo ignora la actividad del sujeto y sobrevalora la mera constatación de leyes comprobadas; a diferencia del positivismo, sus propios hallazgos lo han llevado al papel que cumplen las actividades del sujeto, por tanto, a la necesidad racional (no sólo empírica) de la explicación causal. Desconcertado por el prejuicio que, haciéndolos idénticos, pone juntos a la ciencia y al positivismo, el psicólogo intenta un último argumento. Por supuesto acepta que su trabajo articula la epistemología con la ciencia, pero, paralelamente, enfatiza en que el positivismo constituye una doctrina cerrada de la ciencia. Mientras esta doctrina quiere asignarle unas fronteras cerradas, para los científicos no positivistas la ciencia se encuentra indefinidamente abierta, de igual modo, ella puede abordar cualquier problema siempre y cuando encuentre un método sobre el cual estén de acuerdo los investigadores. Esto último, el acuerdo entre los investigadores, es tal vez la diferencia central para Piaget entre ciencia y filosofía. Dicho llanamente, si bien pueden tener problemas similares, ciencia y filosofía se diferencian en su forma de trabajo. El metafísico trabaja por su cuenta, bajo el ideal del sistema filosófico - de allí que Piaget ubique a la lógica y a la epistemología por fuera de la disciplina filosófica (para ampliar esta afirmación véase Piaget, 1950/1975a, 1950/1975b; Smith, 2006b). Por su parte, el científico confía plenamente en la cooperación racional: su ambición no es el sistema aislado del trabajo de los otros (para una crítica de la noción de sistema filosófico véase especialmente los trabajos de Adorno, 1970/2005; Horkheimer \& Adorno, 1969/1998; Horkheimer, 1947/2010).

A pesar de sus esfuerzos en el arte argumentativo, Piaget fracasó. No pudo convencer a Benrubi. Utilizando un tono con mezcla de sarcasmo, buen humor, y algo de decepción afirma que "No hubo manera; me quedé en positivista, al menos, en este caso, negándome frente a quien me llevaba la contraria creyendo que podría encontrar la verdad con sólo meditar en su despacho a la luz de su genio" (Piaget, 1965/1970, p. 26). Insistimos en que este relato expresa parte de nuestro presente. Cuando encontramos que, al margen de todos sus esfuerzos en contra de tal categoría, Piaget concluye "me quedé en positivista (...)" es difícil evitar pensar que este prejuicio no es privativo de su época. Antes, al contrario, el error que ubica juntos a la ciencia y al positivismo, como si ellos fuesen exactamente la misma práctica, se mantiene casi intacto en la actualidad (véase, Denzin \& Lincoln, 2013). 
Pág 170

Consideramos que los investigadores se encuentran en un doble riesgo: o parten de una concepción implícita de la experiencia; o bien, y en consecuencia, consideran también implícitamente que las teorías son dogmas cerrados que no se dejan someter a la crítica racional. En pocas palabras, si la experiencia sólo sirve para verificar la teoría y si, por su parte, la teoría no se deja someter a la crítica pues no existe ninguna experiencia que la modifique, entonces, y como se mostrará más adelante, la práctica investigativa puede caer en el uso arbitrario de la autoridad.
La vana identidad entre el investigador empírico-analítico y el positivista desconoce que el positivismo llegó a su fin con el empirismo-lógico del Círculo de Viena, a comienzos del pasado siglo (Diéguez, 1998; Klimovsky, 1997; Niiniluoto, Sintonen, \& Wolenski, 2004). Aunque este no es realmente el problema, en contraste, el quid se encuentra en que muchos investigadores confunden un problema propio de la teoría del conocimiento con falsos presupuestos ontológicos. Así, por ejemplo, Dunne, Pryor y Yates (2005), Gutiérrez (2015) y Pérez (1994) no sólo sugieren que la investigación empírico-analítica es positivista, sino que consideran que, para ese tipo de investigación, la realidad es medible y cuantificable. En lógica de proposiciones se diría que de dicha premisa no se puede deducir tal conclusión. Empírico y analítico constituyen una forma de predicar acerca de las dos familias más grandes en la teoría del conocimiento, a saber: empirismo y racionalismo. Esto quiere decir que cuando alguien justifica su estudio desde un punto de vista empírico-analítico está aceptando de entrada que parte tanto de la razón, expresada en sus constructos teóricos, como de la experiencia - por ejemplo, los datos obtenidos- que le sirve para comparar (contrastar) sus asunciones teóricas de partida. Por ningún lado tal expresión predica acerca de la naturaleza de la realidad y, en consecuencia, mucho menos tiene ella que ver con la supuesta cuantificación de la realidad misma. Dicho llanamente, la cuantificación no es un en sí de la realidad, no le pertenece a ella, más bien es uno de los modos a través de los cuales nos aproximamos a la realidad, siempre de forma tentativa.

Ahora bien, otra de las formas de expresar tal prejuicio se hace evidente en el convencimiento, inmodificable por cierto (así como en el caso de Piaget frente a Benrubi), de que las investigaciones teóricas o bien son de carácter cualitativo o bien tienen un escaso valor científico (Chow, 1987). Todo lo anterior nos lleva a formular explícitamente la tesis que se encuentra en los siguientes apartados: la metodología de la investigación debe partir de, y fundamentarse en, la crítica racional. Esta propuesta se apoya en la tradición crítica inaugurada por Kant (1781/2008), de la cual sin duda tanto Piaget cuanto otros científicos son deudores. Al mismo tiempo, problematizamos la forma implícita bajo la cual aparece la noción de experiencia en muchas de las rutas de investigación. Consideramos que los investigadores se encuentran en un doble riesgo: o parten de una concepción implícita de la experiencia; o bien, y en consecuencia, consideran también implícitamente que las teorías son dogmas cerrados que no se dejan someter a la crítica racional. En pocas palabras, si la experiencia sólo sirve para verificar la teoría y si, por su parte, la teoría no se deja someter a la crítica pues no existe ninguna experiencia que la modifique, entonces, y como se mostrará más adelante, la práctica investigativa puede caer en el uso arbitrario de la autoridad.

\section{La crítica como método}

En su análisis del uso indiscriminado y dogmático de la metodología para la investigación en psicología, Delgado (2006) se aproxima a ofrecer una definición del vocablo "psicología" y, por supuesto, de la disciplina a la cual esta palabra se refiere. Quiere hacer evidentes las ambigüedades propias de la psicología que, a nuestro juicio, tienen relaciones con su conformación mixta: ella es tanto una disciplina como una profesión. Así pues, siguiendo su sugerencia, el área de la psicología se encuentra constituida por diferentes grupos de contenidos. A su vez, esta diversidad en sus contenidos origina múltiples orientaciones profesionales, distintas formas de la praxis que se articulan en torno a conceptos y constructos teóricos (véase Delgado, 2008, 2009). Siguiendo a Bakan (1996), la psicología parece existir como una colección de moléculas no relacionadas entre sí. 
Pág 171

La crítica ni dogmática ni escéptica. Ella aparece pues como el camino por medio del cual la razón se somete a su propio tribunal. Ella ocupó el lugar de un camino distinto $y$, nos atrevemos a decir, lo sigue ocupando: desde su nacimiento no ha pretendido convertirse en un medio para adquirir conocimientos sino, si se quiere, en la fundamentación de todo conocimiento posible. Comparada con el dogmatismo, sus pretensiones son menos ambiciosas y, en contraste con el escepticismo, no cede ante la idea, tentadora para algunos, de la imposibilidad del conocimiento.
La ambigüedad práctico-teórica de la disciplina implica aceptar una conclusión evidente para distintos investigadores: la psicología se percibe a sí misma en constante crisis (Bakan, 1996; Castorina, 2002; Driver-Linn, 2003; Mülberge, 2012; Proietto \& Lombardo, 2015; Valsiner, 2006; Vygostsky, 1927/1995; Wieser, 2016). Ahora bien, el diagnóstico común acerca de la crisis de la psicología no se restringe a su carácter disgregado, ya sea en distintos grupos de contenidos teórico-prácticos (Delgado, 2006) o en la ausencia de acuerdos mínimos a nivel conceptual (Bakan, 1996; Castorina, 2002; Driver-Linn, 2003; Mülberge, 2012). También tiene que ver con la presencia de supuestos (casi siempre) implícitos, ya sean antropológicos (Wieser, 2016), metafísicos (Mülberge. 2012), o metateóricos (De Vega, 1981; Overton, 1973, 2006, 2015; Riegel, 1973; Valsiner, 2006; Witherington, Overton, Lickliter, Marshall, \& Narvaez, 2018). Adicionalmente, el diagnóstico hace visible la renuencia a encarar la denominada crisis de la replicabilidad a partir del reconocimiento de un vacío en la existencia de sistemas teóricos acumulativos (Muthukrishna \& Henrich, 2019).

Ahora bien ¿por qué hemos retomado el trabajo de Delgado? ¿Cuál es la importancia de su análisis? A pesar de que para críticos como Gabucio (2006) su texto sólo tiene un carácter de denuncia, a nuestro juicio el valor de sus palabras radica en exponer cómo la actual crisis de la psicología está relacionada fundamentalmente con el uso dogmático de la metodología. Debemos partir de que la metodología cumple una doble función que puede adjetivarse de crítica. Puede argumentarse que la palabra "crítica" tiene aquí al menos dos acepciones: es una propedéutica del conocimiento y un ejercicio ético. En primer lugar, ella cumple la función de una propedéutica del conocimiento, ampliamente teorizada por Kant (1781/2008). Según esa propuesta, la crítica tiene la tarea de explorar las fuentes y los límites del conocimiento y de la experiencia posible; en tal operación, ella debe corregir a la razón de sus propios errores. En su condición de propedéutica, la crítica realiza una especie de purificación de la razón, cierto, pero al mismo tiempo la prepara para encarar de mejor modo el proceso de conocer.

Cuando hablamos de crítica no podemos eludir el hecho de que nos estamos refiriendo a un problema histórico y socialmente situado. Siguiendo una sentencia de Kant (1781/2008), la modernidad puede pensarse como la época del nacimiento de la crítica. Fue justo el filósofo de Königsberg quien se dio a la tarea de despertar a la razón de su sueño dogmático, por un lado, y también del escepticismo que había reaccionado contra la dogmática de la metafísica, por el otro. Si los metafísicos apelaban a un argumento ontológico cuyos predicados de base sugerían que la realidad contaba con propiedades y que, consecuentemente, dichas propiedades se correspondían perfectamente con nuestros estados proposicionales - de allí que asegurasen la existencia fáctica de la idea de Dios-, y si, en su lucha anti dogmática, los escépticos partían de la imposibilidad misma del conocimiento; entonces, a pesar de todo ello Kant alzó su propuesta en contra de estas dos formas de la teoría del conocimiento. La crítica ni dogmática ni escéptica. Ella aparece pues como el camino por medio del cual la razón se somete a su propio tribunal. Ella ocupó el lugar de un camino distinto y, nos atrevemos a decir, lo sigue ocupando: desde su nacimiento no ha pretendido convertirse en un medio para adquirir conocimientos sino, si se quiere, en la fundamentación de todo conocimiento posible. Comparada con el dogmatismo, sus pretensiones son menos ambiciosas y, en contraste con el escepticismo, no cede ante la idea, tentadora para algunos, de la imposibilidad del conocimiento. No obstante, debe insistirse, su utilidad es puramente negativa, pues ella "No serviría para ampliar nuestra razón, sino sólo para clarificarla y preservarla de errores, con lo cual se habría adelantado ya mucho" (Kant, 1781/2008, p. 58). 
Pág 172

La utilidad de la crítica es negativa porque, más que lanzarnos rápidamente hacia la conquista de un nuevo conocimiento, con ella somos conscientes de que debemos primero aclarar nuestros presupuestos de partida y, por ello, depurar nuestros errores. En una perspectiva actual esto significa que, sin la crítica de nuestras teorías, y sin la corrección de nuestras metodologías, la investigación no podría ser más que la aplicación de fórmulas preconcebidas.
Entiéndase bien, la utilidad de la crítica es negativa porque, más que lanzarnos rápidamente hacia la conquista de un nuevo conocimiento, con ella somos conscientes de que debemos primero aclarar nuestros presupuestos de partida y, por ello, depurar nuestros errores. En una perspectiva actual esto significa que, sin la crítica de nuestras teorías, y sin la corrección de nuestras metodologías, la investigación no podría ser más que la aplicación de fórmulas preconcebidas. Un ejemplo de la crítica rigurosa a los propios constructos teóricos lo encontramos en el trabajo de Pérez-Echeverría, Mateos, Pozo y Scheuer (2001). Estos autores revisan diferentes concepciones sobre el constructivismo, señalan los excesos en el uso del concepto y, además, muestran el error que supone atribuir características constructivistas a procesos de adquisición de conocimiento que carecen de propiedades constructivas. Así pues, el ejercicio de la negatividad nos previene frente a un tipo de praxis cognoscitiva incapaz de separarse del sentido común.

Su segundo sentido indica que sólo por medio de la crítica puede darse respuesta al interrogante popperiano acerca de cómo detectar y eliminar nuestros errores teóricos y, cabe añadir, cognoscitivos. Aclaremos lo anterior: Popper (1972/1991) ha mostrado la insuficiencia de la vieja pregunta por las fuentes del conocimiento. Una pregunta que, a pesar de ser antigua, sigue siendo utilizada en nuestros días. Desde su punto de vista, el programa de rastrear el conocimiento hasta sus fuentes últimas, sean ellas empíricas o lógico-racionales, no puede nunca realizarse. Concentrarse en el origen del conocimiento conlleva una regresión al infinito según la cual siempre que justificamos algo tenemos que justificar los medios de la justificación. Lógicamente, no existe ningún fundamento último para nuestras proposiciones, ni en la experiencia ni en la razón. Así pues, si bien existe una variedad de fuentes del conocimiento, siguiendo a Popper, todas ellas carecen de autoridad. Esto también significa que, al hacer énfasis en ellas, la teoría filosófica de las fuentes del conocimiento comete un error: no diferencia con suficiente claridad entre cuestiones de origen y cuestiones de validez. Partiendo de la premisa de que no existen fuentes ideales del conocimiento y de que, consecuentemente, los tipos mismos de fuentes nos pueden llevar al error, Popper propone una pregunta que no se concentra en el origen del conocimiento sino, más precisamente, en su refutabilidad. Tal interrogante es: “(...) ¿Cómo podemos detectar y eliminar el error?" (Popper, 1972/1991, p. 49). El contenido proposicional de la respuesta a dicha cuestión reza: "Criticando las teorías y presunciones de otros y -si podemos adiestrarnos para hacerlo- criticando nuestras propias teorías y presunciones" (p. 50). Popper sugiere que, a pesar de ser sumamente deseable, la última parte del enunciado no es necesaria debido a que, si nosotros mismos no lo hacemos, otros pueden estar dispuestos a criticar nuestras teorías.

De esta segunda acepción se desprende que la crítica es a la vez una actividad gnoseológica y un principio ético. Popper (1972/1991) ubica los orígenes del racionalismo crítico en la antigüedad clásica, y por ello expresa que su concepto de crítica no coincide completamente con la propedéutica kantiana del conocimiento; según dice, Kant no fue capaz de articular el juicio sobre el conocimiento con su propia teorización acerca de los fundamentos racionales de la moralidad humana (Kant 1788/2000,1785/2012). En contraste, el racionalista crítico considera que existe una relación entre conocimiento y ética. Si el principio kantiano de autonomía sostiene que, por elevada que ella sea, no debemos aceptar la orden de ninguna autoridad en cuestiones de ética, y esto equivale a afirmar nuestra capacidad de tomar distancia de toda autoridad; entonces, la crítica no sólo se dirige en contra del error lógico (o metodológico) sino que, por ello mismo, nos mantiene alerta sobre los posibles excesos éticos inmanentes a la investigación científica o académica (para una revisión 
Pág 173

La crítica se muestra con un doble rostro: de una parte, y en su condición de propedéutica, prepara a la razón para el ejercicio metódico del conocimiento; de la otra, además de querer corregir nuestros errores teóricos, lógicos y, agreguemos, metodológicos, la crítica cumple también una función moral, es decir, nos previene frente a la creencia ciega en la autoridad y, paralelamente, nos muestra que todo conocimiento debe partir de la aceptación de la propia ignorancia. acerca del creciente control social por parte de las autoridades institucionales sobre "la ética de la investigación" véase Valsiner, 2006). Una afirmación científica, tenga la forma de una hipótesis, de un supuesto o, incluso, de un enunciado observacional, no es verdadera porque haya sido dicha por alguna autoridad. Es verdadera, aunque incierta y tentativa, porque el rigor teórico-metodológico nos permite afirmarla únicamente en cuanto conjetura. Lejos de ser absolutas, como son, por ejemplo, las creencias dogmáticas y religiosas, las afirmaciones científicas son provisionales, y se encuentran sometidas a la caducidad.

Popper (2001) insiste en la relación entre ética y conocimiento. Presenta algo que, a primera vista, pudiera parecer una simple aporía, una sin salida lógica. Esto es, se encarga de justificar racionalmente "el conocimiento de la ignorancia". ¿Cómo es posible poner juntos conocimiento e ignorancia? Actualizando la enseñanza de Sócrates y, con ella, reavivando nuestra memoria intelectual, encuentra que la relación entre conocimiento e ignorancia no sólo es posible, sino que es moralmente necesaria. A partir de la clásica expresión socrática "sólo sé que no sé nada" (Platón. 1985, 21d-e), y de su pertinencia para el mundo contemporáneo, plantea que esa relación entre conocimiento y ética comienza por la aceptación de que, en no pocos sentidos, somos ignorantes. Si queremos una teoría del conocimiento, dice, necesitamos comenzar por admitir nuestra propia ignorancia. El conocimiento de nuestra propia ausencia de conocimiento hace visibles de entrada, y críticamente, nuestros límites. Dicho de otro modo, el conocimiento científico se encuentra siempre limitado; "Consiste en conjeturas comprobables -el mejor de los casos-, conjeturas que han sido objeto de las más duras pruebas, conjeturas inciertas" (Popper, 2001, p. 2). Conjeturas inciertas es un modo de decir - renovando a Sócrates-que no hay conocimiento absoluto cuando se habla de ciencia. Ella es sólo un conocimiento conjetural o hipotético.

Para terminar, la crítica se muestra con un doble rostro: de una parte, y en su condición de propedéutica, prepara a la razón para el ejercicio metódico del conocimiento; de la otra, además de querer corregir nuestros errores teóricos, lógicos y, agreguemos, metodológicos, la crítica cumple también una función moral, es decir, nos previene frente a la creencia ciega en la autoridad y, paralelamente, nos muestra que todo conocimiento debe partir de la aceptación de la propia ignorancia. Estos presupuestos han sido llevados por el pragmatismo de Rorty (1991/1996) más allá de toda epistemología, y de toda concepción prescriptiva del conocimiento. Este autor ha dicho que además de consistir en criterios especificables de antemano, ya sea por correspondencia o por coherencia, la verdad es también aquello que una comunidad decide como bueno para ella misma. La ciencia, en este sentido, es sobre todo solidaridad. Y la racionalidad, aquella facultad por intermedio de la cual es posible la crítica, puede tener al menos dos sentidos: ser racional es ser metódico, ello es, tener criterios fijados de antemano; parte de nuestra noción de crítica es metódica, contiene ella misma criterios. En su segundo significado, ser racional es ser sensato o razonable; la otra parte de nuestra noción de crítica, sobre todo en su acepción moral, puede ser derivada de este concepto de racionalidad. Dicho llanamente, racionalidad aquí:

Designa un conjunto de virtudes morales: tolerancia, respeto a las opiniones de quienes nos rodean, disposición a escuchar, recurso a la persuasión antes que a la fuerza. Éstas son las virtudes que deben poseer los miembros de una sociedad civilizada para que ésta dure (Rorty, 1991/1996, p. 59). 
Debe insistirse en que estas formas de la racionalidad bien pueden articularse a la crítica en sus dos acepciones. Como propedéutica, ella parte necesariamente de criterios rigurosos, es decir, ella ejerce como el fundamento del conocimiento posible; hace visibles, por ejemplo, los límites de las capacidades cognitivas con las cuales hemos sido dotados en tanto que especie. Bajo su forma ética, siguiendo la sugerencia de Rorty, la crítica señala el límite de nuestros argumentos: nos muestra que, independientemente de si participamos de ellas, existen otras formas posibles de acercarse al conocimiento. Limita pues el alcance de nuestras creencias teóricas permitiendo así que nos acerquemos a las de otros. Esto no supone abandonar los propios presupuestos, aunque sí implica que seamos capaces de saber que otros presupuestos pueden existir y que, de hecho, lo hacen, existen (Piñeres-Sus, 2017). Así como en la vida social podemos criticar todas aquellas nociones normativas que, por doquier, buscan prescribir reglas estrictas para la existencia, así como los grupos y movimientos sociales pueden no estar de acuerdo con aquellos criterios culturales que les indican lo que ellos deben ser (Butler, 2002), asimismo, insistimos, en su carácter de éticamente insumisa, la crítica del conocimiento devela la urgencia de abrir nuestros marcos cognoscitivos.

\section{Breve apunte sobre teoría y experiencia}

Se había dicho que, además de proponer la crítica como fundamento metodológico de la investigación en psicología, en este artículo pretendemos cuestionar esa forma implícita bajo la cual aparecen las nociones de experiencia y de teoría. En lo que respecta a la psicología, la relación entre teoría y experiencia ha sido problematizada desde finales del siglo XIX. De acuerdo con las investigaciones de Mülberge (2012), Proietto y Lombardo (2015) y Wieser (2016), parte del diagnóstico de la crisis de la disciplina tuvo su centro en el antagonismo entre: por un lado, la concepción de una experiencia auténtica - propuesta por Willy en 1908-, según la cual el yo y el ambiente no son entidades distintas, sino que son componentes de un complejo total definido como "la intimidad del mundo externo personalizado" (the intimacy of the personalized external world) (Mülberge, 2012, p. 434); y, por el otro, la postura aún metafísica de teóricos como Wundt quienes, a pesar de haber desarrollado métodos experimentales y de haber introducido las investigaciones psicofísicas, seguían manteniendo una concepción dualista de la experiencia, dividida en causalidad física y causalidad mental.

En contraste, para Willy la experiencia debía ser considerada como experiencia total; propuso una definición holística del ser humano que lo consideraba como una especie psicofísicamente indivisible (Mülberge, 2012). Así, por ejemplo, no solo se distanció del psicólogo de Leipzig sino también de su mentor, el filósofo Avenarius. Según su opinión, este último sostuvo una concepción de la experiencia fundamentalmente empírica, aunque susceptible de ser sometida a la crítica. Por su parte, a partir de un interés estético, Willy (1896, p. 59) llegó a proponer la fórmula "todo es experiencia y experiencia es todo" (como se citó en Mülberge, 2012). No en vano introdujo un término de difícil traducción al castellano, a saber: Natürliche Welterfahrung; más que tratarse de una experiencia empírica controlada experimentalmente, al ser estética esa experiencia natural del mundo es ante todo experiencia sensible.

La disyunción entre experiencia o teoría es un elemento vinculado con la crisis de la psicología. En su diagnóstico, Valsiner (2006) ha insistido en que los investigadores han estado más interesados en arrojar datos que en producir teoría; mientras que, en un sentido similar, Bakan (1996) ha afirmado que, si bien existe una proliferación de artículos y de conferencias, la psicología reciente se ha desviado del propósito de 
Pág 175

Aunque todos sabemos que las ciencias sociales, incluida la psicología, son disciplinas empíricas que combinan teoría y experiencia, y aunque es por nosotros conocido que los grandes investigadores de nuestra área tienen clara esta conformación mixta y que, en tal sentido, conocen el justo lugar de ambos dominios lógicos, no por ello podemos dejar de cuestionar el lugar ocupado por teoría y experiencia en algunos de nuestros debates y, ciertamente, en la investigación. formular teorías. Por el contrario, los investigadores se han concentrado en mostrar los resultados de sus estudios particulares. La metáfora aquí es la de un archivo de datos desprovisto de una orientación teórico-sistemática.

Si conciben de modo unilateral las relaciones entre teoría y experiencia, es posible pensar con $\underline{\text { Valsiner (2006) }}$ que algunos investigadores parecen pasar por alto el ciclo metodológico, esto es, las relaciones intrínsecas entre las facetas teóricas y empíricas del proceso de investigación. De acuerdo con el análisis de Valsiner, la psicología se ha dividido por su fijación metodológica, olvidando incluso preguntas fundamentales que conducen a la creación de conocimiento básico. Así las cosas, es frecuente leer debates metodológicos que se concentran en - al menos - tres antinomias mencionadas por diversos autores, a saber: investigación cualitativa/investigación cuantitativa (Ato, López, \& Benavente, 2013; Bogdan \& Biklen, 2007; Cosgrove, Wheeler, \& Kosterina, 2015; Gough, 2015; Mouton, 1996; Parker, 1997, 2005, 2015; Valsiner, 2013); investigación empírica/investigación teórica (González, 2002; Piaget, Lazarsfeld, \& Mackenzie, 1976; Sternberg, 1996); investigación básica/investigación aplicada (Ato, López, \& Benavente, 2013; Fathalla \& Fathalla, 2008; Rivière, 1990).

No sin prejuicios, quienes investigan en las denominadas ciencias sociales intentan defender, cuando no imponer, su propia concepción de la metodología. Unos y otros se encuentran convencidos de que sus formas de acercarse a los problemas del conocimiento y, en estrecha conexión, los criterios de verdad que utilizan en su práctica investigativa son más correctos, y por ello menos falibles, de los que defienden aquellos que consideran sus "adversarios" metodológicos. Algunas voces concentran sus propias creencias en el viejo problema de las fuentes del conocimiento: para unos, los datos adquieren el carácter de entidades autónomas, sobre todo si encuentran su fundamento último en la experiencia controlada; mientras que, para otros, la experiencia vivida de las personas funge como el fundamento último, incuestionado y autoevidente de toda investigación (para una crítica de la noción de experiencia vivida véase Benjamin, 2001, 2011).

Aunque todos sabemos que las ciencias sociales, incluida la psicología, son disciplinas empíricas que combinan teoría y experiencia, y aunque es por nosotros conocido que los grandes investigadores de nuestra área tienen clara esta conformación mixta y que, en tal sentido, conocen el justo lugar de ambos dominios lógicos, no por ello podemos dejar de cuestionar el lugar ocupado por teoría y experiencia en algunos de nuestros debates y, ciertamente, en la investigación. Se nos dice, por ejemplo, que para los investigadores cuantitativos las teorías deben ser claras para poder compararlas con los datos; no se pueden comparar datos obtenidos a partir de la operacionalización de una teoría en sus variables con las variables obtenidas de otra teoría, por supuesto. También se nos dice que la teoría en la investigación cualitativa es multicíclica y, como es de esperarse, no puede estar dada de una vez y para siempre. Hasta aquí no parece haber ningún problema. Muy seguramente, la mayoría de nosotros estamos de acuerdo con ambas afirmaciones. Sin embargo, y partiendo de la idea de que son verdaderas, tales aserciones no dejan de dar la impresión de tratarse en ocasiones de fórmulas poco reflexionadas.

Pues bien, esta propuesta puede plantearse del siguiente modo: frecuentemente, los investigadores entienden la experiencia simplemente como ilustración —en el sentido de ejemplificación- de sus creencias teóricas; no como aquello que, además de la razón, nos enseña, y nos permite criticar nuestras teorías. En múltiples ocasiones buscamos acomodar las teorías a todos los datos posibles. Pero también revela que 
Hemos intentado oponernos conceptualmente a las visiones parcializadas y unilaterales. ¿Teoría o experiencia? En tal premisa el error es la disyunción. El énfasis en la experiencia muda, es la ausencia de experiencia; el énfasis en la teoría acrítica, es la negación del conocimiento mismo. Dicho de otra manera, cuando confiamos demasiado en nuestras teorías, y las vemos más grandes de lo que son, nos queda únicamente confirmarlas por doquier. continuamente buscamos argumentos de autoridad para preservarnos a nosotros mismos. Cuando forzamos que toda experiencia sea confirmatoria de nuestros presupuestos teóricos es porque, lógicamente, poco nos importa la experiencia: ella sólo sirve para ilustrar lo conocido de antemano.

La otra parte del argumento sugiere una relación de identidad entre teoría y dogma. Siempre que la teoría propende por ser verificada (o ilustrada) por todos aquellos casos hallados en su camino, ella deviene inevitablemente en una posición dogmática. En suma, el lugar dogmático para el cual ni la experiencia ni, finalmente, la teoría nos enseñan, pues no puede haber enseñanza cuando el aprendiz cree saberlo todo y no es capaz de moverse de su lugar.

\section{Conclusiones}

Como la memoria, en este caso el epílogo se encuentra irremediablemente abierto, no existen en él afirmaciones concluyentes. La relación entre metodología, crítica, teoría y experiencia, esas cuatro categorías que componen esta reflexión en su totalidad, expresan nuestra preocupación académica y sincera por la disciplina psicológica, y por sus formas de investigación. Seguramente como a algunos, nos inquieta aquel dogmatismo en el que es tan fácil creer. Aunque, tal vez como a otros, nos importa que las investigaciones se sigan haciendo con su rigor característico. Pero ese rigor indudablemente tiene que ser practicado a nivel empírico y teórico. La conjunción aquí no es simple contingencia, es una necesidad lógica y, de acuerdo con lo dicho en estas páginas, ética. Separados, estos ámbitos no pueden funcionar más que de modo incompleto, parcial. Hemos intentado oponernos conceptualmente a las visiones parcializadas y unilaterales. ¿Teoría o experiencia? En tal premisa el error es la disyunción. El énfasis en la experiencia muda, es la ausencia de experiencia; el énfasis en la teoría acrítica, es la negación del conocimiento mismo. Dicho de otra manera, cuando confiamos demasiado en nuestras teorías, y las vemos más grandes de lo que son, nos queda únicamente confirmarlas por doquier.

\section{Referencias}

Adorno, Th. (1970/2005). Dialéctica negativa. La jerga de la autenticidad. Obra completa 6. Madrid: Akal.

Ato, M., López, J. J., \& Benavente, A. (2013). Un sistema de clasificación de los diseños de investigación en psicología. Anales de psicología 23(3), 1038-1059. doi: https:// doi.org/10.6018/analesps.29.3.178511

Bakan, D. (1996). The Crisis in Psychology. Journal of Social Distress and the Homeless, 5(4), 335-342. doi: https://doi.org/10.1007/BF02092909

Benjamin, W. (1936/2011). El narrador. En W. Benjamin, Iluminaciones IV. Para una crítica de la violencia y otros ensayos (pp. 125-152). Montevideo: Aguilar.

Benjamin, W. (1939/2001). Sobre algunos temas en Baudelaire. En W. Benjamin, Ensayos escogidos (pp. 7-41). México D.F.: Coyoacán.

Beth, E. W., \& Piaget, J. (1961/1980). Epistemología, matemática y psicología. Relaciones entre la lógica formal y el pensamiento real. Barcelona: Crítica.

Bogdan, R. C., \& Biklen, S. K. (2007). Qualitative research for education: An introduction to theory and methods, 5th ed. Boston: Allyn \& Bacon.

Butler, J. (2002). What is critique? An essay on Foucault's virtue. En D. Ingram, The political: Blackwell readings in continental philosophy (pp. 212-226). Malden, MA: Blackwell.

Castorina, J. A. (2002). El impacto de la filosofía de la escisión en la psicología del desarrollo cognoscitivo. Psykhe, 11(1), 15-27. 
Chow, S. L. (1987). Meta-Analysis of Pragmatic and Theoretical Research. A Critique. The Journal of Psychology: Interdisciplinary and Applied, 121(3), 259-271. doi: http://dx.doi.org/10.1080/00223980.1987.9712666

Cosgrove, L., Wheeler, E., \& Kosterina, E. (2015). Quantitative methods: science means and ends. En I. Parker (Ed.), Handbook of critical psychology (pp. 15-23). New York: Routledge.

Daniels, H., Cole, M., \& Wertsch, J. V. (2007). The Cambridge Companion to Vygotsky. Cambridge: Cambridge University Press.

De Vega, M. (1981). Una exploración de los metapostulados de la psicología contemporánea: el logicismo. Análisis y modificación de conducta, 7(16), 345-375.

Delgado, J. (2006). Psicología en crisis. Metodología dogmática. Encuentros y desencuentros. Anuario de Psicología, 37(1 y 2), 7-26.

Delgado, J. (2008). Las difíciles relaciones entre teoría, práctica y metodología en la psicología del siglo XXI. (I) Sobre algunas disyuntivas. Anuario de psicología, 39(3), 387-409.

Delgado, J. (2009). Las difíciles relaciones entre teoría, práctica y metodología en la psicología del siglo XXI. (II) Propuesta metodológica. Anuario de psicología, 40(1), 117-133.

Denzin, N. K., \& Lincoln, Y. S. (Coords). (2013). Las estrategias de investigación cualitativa. Barcelona: Gedisa.

Diéguez, A. (1998). Realismo científico. Una introducción al debate actual en filosofía de la ciencia. Málaga: Servicio de Publicaciones e Intercambio Científico de la Universidad de Málaga.

Driver-Linn, E. (2003). Where is psychology going? Structural fault lines revealed by psychologists' use of Kuhn. American Psychologist, 58(4), 269-278. doi: doi:10.1037/0003-066X.58.4.269

Dunne, M., Pryor, J., \& Yates, P. (2005). Becoming a researcher. A research companion for the social sciences. Poland: Open University Press.

Fathalla, M. F., \& Fathalla, M. M. F. (2008). Guía práctica de investigación en salud. Washington: Organización Panamericana de la Salud.

Feyerabend, P. (2003). Tratado contra el método. Madrid: Tecnos.

Gabucio, F. (2006). No hay metodología sin epistemología. Sobre crisis y dogmatismos. Anuario de Psicología, 37(1 y 2), 45-52.

González, M. F. (2010). ¿Pueden los clásicos decir algo nuevo sobre la identidad? Una revisión de las ideas de Bakhtin, Vygotsky y Mead en tiempos de identidad líquida. Estudios de psicología, 31(2), 187-203.

González, Ó. L. (2002). Las ciencias cognitivas y su fundamentación filosófica (Tesis Doctoral). Universidad Complutense de Madrid, Madrid.

Gough, B. (2015). Qualitative methods: critical practices and prospects from a diverse field. En I. Parker (Ed.), Handbook of critical psychology (pp. 107-116). New York: Routledge.

Gutiérrez, E. (2015). A propósito de los "falsos opuestos" en Ciencias Sociales -La IAP como posibilidad para la lectura integral y crítica de la realidad. Campos, 3(2), 261-279.

Horkheimer, M. (1947/2010). Crítica de la razón instrumental. Madrid: Trotta.

Horkheimer, M., \& Adorno, Th. (1969/1998). Dialéctica de la ilustración. Fragmentos filosóficos. Madrid: Trotta.

Kant, I. (1781/2008). Crítica de la razón pura. México D.F.: Taurus.

Kant, I. (1785/2012). Fundamentación para una metafísica de las costumbres. Madrid: Alianza.

Kant, I. (1788/2000). Crítica de la razón práctica. Madrid: Alianza. 
Klimovsky, G. (1997). Las desventuras del conocimiento científico. Una introducción a la epistemología. Buenos Aires: A-Z Editora.

Kuhn, Th. (1962/2013). La estructura de las revoluciones científicas. México D.F.: Fondo de Cultura Económica.

Mouton, J. (1996). Basic concepts in the methodology of the social sciences. Pretoria: HSRC Publishers.

Mülberge, A. (2012). Wundt contested: The first crisis declaration in psychology. Studies in History and Philosophy of Biological and Biomedical Sciences 43, 434-444. doi: https://doi.org/10.1016/i.shpsc.2011.11.002

Müller, U., Carpendale, J. I. M., \& Smith, L. (Eds.). (2009). The Cambridge Companion to Piaget. New York: Cambridge University Press.

Muthukrishna, M., \& Henrich, J. (2019). A problem in theory. Nature Human Behavior, 3, 221-229. doi: https://doi.org/10.1038/s41562-018-0522-1

Niiniluoto, I., Sintonen, M., \& Wolenski, J. (Eds.). (2004). Handbook of Epistemology. Dordrecht, FIDH: Springer.

Overton, W. F. (1973). On the Assumptive Base of the Nature-Nurture Controversy-Additive versus Interactive Conceptions. Human Development, 16(1-2), 74-89. doi: https:// doi.org/10.1159/000271268

Overton, W. F. (1990). Reasoning, necessity, and logic: Developmental perspectives. Hillsdale: Erlbaum.

Overton, W. F. (2006). Developmental psychology: Philosophy, Concepts, Methodology. En R. M. Lerner, (Ed.), Handbook of child psychology. Vol 1 Theoretical models of human development (pp. 18-88). New Jersey: Wiley.

Overton, W. F. (2015). Processes, relations, and relational-developmental-systems. En R. M. Lerner, W. Overton, \& P. C. M. Molenaar (Eds.), Handbook of child psychology and developmental science. Vol 1. Theory and method (pp. 9-62). New Jersey: Wiley.

Parker, I. (1997). Qualitative research. En P. Banister, E. Burman, I. Parker, M. Taylor \& C. Tindall (Eds.), Qualitative methods in psychology. A research guide (pp. 1-16). Bristol: Open University.

Parker, I. (2005). Qualitative psychology. Introducing radical research. New York: Open University.

Parker, I. (2015). Psychology after discourse analysis. Concepts, methods, critique. London: Routledge.

Pérez-Echeverría, M., Mateos, M., Pozo, J. I., \& Scheuer, N. (2001). En busca del constructivismo perdido: concepciones implícitas sobre el aprendizaje. Estudios de Psicología, 22(2), 155-173. doi: https://doi.org/10.1174/021093901609479

Pérez, G. (1994). Investigación cualitativa. Retos e interrogantes. Madrid: La Muralla S.A.

Piaget, J. (1950/1975a). Introducción a la epistemología genética. Vol. 2. El pensamiento físico. Buenos Aires: Paidós.

Piaget, J. (1950/1975b). Introducción a la epistemología genética. Vol. 3. El pensamiento biológico, psicológico y sociológico. Buenos Aires: Paidós.

Piaget, J. (1957). Logic and psychology. New York: Basic Books.

Piaget, J. (1965/1970). Sabiduría e ilusiones de la filosofía. Barcelona: Península.

Piaget, J. (1971/1977). Ensayo de lógica operatoria. Buenos Aires: Editorial Guadalupe.

Piaget, J., Lazarsfeld, P. F., \& Mackenzie, W. J. M. (1976). Tendencias de la investigación de las ciencias sociales. Madrid: Alianza.

Piñeres-Sus, J. D. (2017). Lo humano como ideal regulativo. Imaginación antropológica: cultura, formación y antropología negativa. Medellín: Fondo Editorial FCSH, Universidad de Antioquia.

Platón. (1985). Apología. Madrid: Gredos. 
Popper, K. (1972/1991). Conjeturas y refutaciones. El desarrollo del conocimiento científico. Barcelona: Paidós.

Popper, K. (2001). El conocimiento de la ignorancia. Polis, Revista de la Universidad Bolivariana, 1(1), 1-5.

Proietto, M., \& Lombardo, G. P. (2015). The "crisis" of psychology between fragmentation and integration: The Italian case. Theory \& psychology, 25(3), 313-327. doi: https://doi.org/10.1177/0959354315578726

Riegel, K. F. (1973). An Epitaph for a Paradigm. Human Development, 16(1-2), 1-7. doi: https://doi.org/10.1159/000271262

Rivière, Á. (1985). La psicología de Vygotsky. Madrid: Visor.

Rorty, R. (1991/1996). Objetividad, relativismo y verdad. Escritos filosóficos I. Barcelona: Paidós.

Smith, L. (2006a). Norms in human development: Introduction. En J. Vonèche \& L. Smith, (Eds.), Norms in Human Development (pp. 1-31). New York: Cambridge University Press.

Smith, L. (2006b). Norms and normative facts in human development. En J. Vonèche \& L. Smith, (Eds.), Norms in Human Development (pp. 103-137). New York: Cambridge University Press.

Sternberg, R. J. (1996). Investigar en psicología. Una guía para la elaboración de textos científicos dirigida a estudiantes, investigadores y profesionales. Barcelona: Paidós.

Tryphon, A., \& Vonèche, J. (1996/2000). Piaget-Vygotsky: la génesis social del pensamiento. Buenos Aires: Paidós.

Valsiner, J. (2006). Dangerous Curves in Knowledge Construction within Psychology. Fragmentation of Methodology. Theory \& Psychology, 16(5), 597-612.

Valsiner, J. (2013). A guided science. History of psychology in the mirror of its making. New Brunswick: Transaction Publishers.

Van der Veer, R. (2014). Lev Vygotsky. London: Bloomsbury Academic.

Wieser, M. (2016). Psychology's "Crisis" and the Need for Reflection. A Plea for Modesty in Psychological Theorizing. Integrative Psychological and Behavioral Science, 50(3), 359-367. doi: https://doi.org/10.1007/s12124-016-9343-9

Witherington, D., Overton, W., Lickliter, R., Marshall, P., \& Narvaez, D. (2018). Metatheory and the Primacy of Conceptual Analysis in Developmental Science. Human Development, 61(3), 181-198. doi: https://doi.org/10.1159/000490160

Yasnitsky, A., \& Van der Veer. R. (2016). Revisionist revolution in Vygotsky studies. London: Routledge.

Yasnitsky, A., Van der Veer, R., \& Ferrari, M. (Eds.). (2014). The Cambridge handbook of cultural-historical psychology. Cambridge: Cambridge University Press.

Zavershneva, E., \& Van der Veer, R. (2018). Vygotsky's Notebooks: A selection. Singapore: Springer. 